\title{
Developing Higher Quality Conductors and Insulators to Enable Fieldable FIB edits to Complex Microelectronics
}

Peter Lewis, Isaac Moran, Elizabeth Brundage, Robert Day and Katherine Graham

Draper, United States

Materials deposition in focused ion beam (FIB) systems enables direct-write 'bottoms-up' fabrication of sub-micron features. In particular, such technology has the potential to impact the creation and editing of small electronic devices. However, the material quality of both dielectrics and conductors, and thus the electrical performance, is low due to the impurities left behind after deposition. In a traditional Ga FIB, gallium implantation may foul both the deposited material and the adjacent dielectric, causing performance and reliability concerns. To help counter this effect, we sought to explore new FIB based plasma deposition chemistries utilizing less characterized ion sources as well as novel precursors. Ultimately we would like to develop new FIB capabilities that would enable rapid circuit editing for fieldable microelectronic systems.

Thus far, our work has focused on exploring plasma FIB deposition with multiple organometallic precursors and 4 ion types: $\mathrm{Xe}, \mathrm{O}$, Ar, and $\mathrm{N}$. Commercially available FIB precursors, tungsten hexacarbonyl and trimethyl(methylcyclopentadienyl)platinum(IV), were analyzed along with more novel FIB chemistries for gold and copper deposition. A Thermofisher Helios Hydra DualBeam was used for the work with enhancements to enable gas co-flow during deposition with the goal of reducing the carbon content of the FIB deposited material and thus increasing its purity. Precursor deposition is evaluated and analyzed over a variety of deposition conditions aimed at finding highest purity and best performance materials. Automated scripts to control deposition were developed to run these procedures and enable faster analysis of the parameter space. The structural morphology of the deposited materials were characterized by analysis of scanning electron micrographs along with profilometry and optical tomography. Additionally EDS elemental analysis, and electrical measurements were used to assess deposition composition. 